Ann. Biol. anim. Bioch. Biophys., 1977, 17 (6), 971-985.

\title{
Machine à traire : paramètres physiques caractérisant le fonctionnement du manchon trayeur
}

\author{
par J. LE DU \\ avec la collaboration technique de J. L. MOHAER ef P. LAMBION
}

Laboratoire de Recherches sur la Traite, I. N. R. A., E. N. S. A., 65, rue de Saint-Brieuc 35042 Rennes Cedex.

Summary. The milking machine : physical parameters characterizing teat cup liner working conditions.

The working conditions of the teat cup liner for cows were studied in the laboratory by simulating different milking conditions at 3 liquid flow rates though the milking cluster $(0 \mathrm{l} / \mathrm{min}, 1 \mathrm{l} / \mathrm{min}$ and $4 \mathrm{l} / \mathrm{min})$ for different milk pipeline heights, air admissions at the claw and types of liners. Pulsation, vacuum under the teat and liner wall movement were recorded simultaneously (fig. 3). The results show that :

1) Milk flow rate was the main factor changing the basic working conditions of the liner. It decreased the speed of liner wall movement (fig. 7) and increased vacuum fluctuation (fig. 5). The mean vacuum under the teat was affected (fig. 6a) and the time during which the liner remained open was modified (fig. 6b).

2) Air admission at the claw reduced these disturbances and was an important regulation factor, whatever the height at which the milk was evacuated (fig. 5, 6, 7, 8).

3) With the low line, the mean vacuum under the teat end was highter than with the high line (fig. 6a). However, as maximum and minimum vacuums increased simultaneously, vacuum (fig,. $4 a, b$ ) fluctuations remained almost as important as with a high line (fig. 4c). 4) During milk flow, the time during which the liner remained open increased when the mean vacuum under the teat end decreased (fig. 6). Thus, the movement of the liner could not be deduced from the pulsation curve.

Considering the multiple interactions between the various physical parameters of the milking machine, it would appear too early to define optimal working conditions in too much detail until the physiological requirements of the animal are better understood.

\section{Introduction.}

Le rôle du manchon trayeur, seul composant de la machine à traire en contact avec l'animal, est déterminant en ce qui concerne la vidange de la mamelle. La compréhension de son fonctionnement nécessite l'étude des forces appliquées au trayon par son intermédiaire. Le niveau du vide sous le trayon est mesurable à l'aide de capteurs de pression à jauges (Noorlander ef Schalm, 1958) et l'enregistrement direct du mouvement des parois du manchon par des capteurs de déplacement miniaturisés est devenu possible (Thiel ef al., 1968). 
Les recherches entreprises à cet objet ont notamment montré que :

- les fluctuations importantes du vide sous le trayon durant l'écoulement du lait (Thiel, Clough et Akam, 1964 ; Witzel et McDonald, 1964) auraient, suivant de nombreux auteurs cités par Fell (1964) et par Nyhan (1968), un effet néfaste sur le plan sanitaire et ralentiraient la traite. De plus, Thiel et al. (1969) et Whittlestone et Jones (1969) ont démontré expérimentalement que ce vide fluctuant peut favoriser la pénétration, par le canal du trayon, de germes pathogènes dans la mamelle.

- un niveau de vide élevé sous le trayon, considéré en général comme bénéfique pour la rapidité de traite et la stimulation perçue par l'animal, aurait sur le plan sanitaire les mêmes inconvénients qu'un vide instable (Revue bibliographique de Labussière et Richard, 1965).

- Le mouvement des parois du manchon a paradoxalement assez peu retenu l'attention (Thiel, Clough et Akam, 1964 ; O'Callaghan, 1974).

Or, l'opinion générale est que la décongestion du trayon dépendrait de la pression appliquée par le manchon lors de la phase de fermeture, du reste qualifiée de « massage» (Zaks, 1962 ; Thiel, 1968). En outre, le manchon, par le rythme de déplacement de ses parois et les variations de la pression qu'il applique au trayon, serait l'un des facteurs principaux de déclenchement du réflexe d'éjection du lait (Zaks, 1962 ; Tröger et Lohr, 1967 ; Brandsma, 1968).

Dans ce contexte, nous avons étudié l'incidence des quatre facteurs suivants sur le vide sous le trayon et le mouvement du manchon trayeur:

1. Le débit du lait à travers le manchon,

2. L'admission d'air à la griffe,

3. La hauteur du lactoduc au-dessus du quai,

4. Le type de manchon-trayeur.

\section{Matériel et méthodes.}

Installation de traite $\left(^{(1)}\right.$.

L'installation de traite expérimentale utilisée est constituée d'un lactoduc ( $\mathrm{di}=34 \mathrm{~mm}, \mathrm{I}=4,40 \mathrm{~m}$ ) relié à une chambre de réception d'une contenance de 23 I connectée à une pompe à vide d'une capacité de $500 \mathrm{NI} / \mathrm{mn}\left({ }^{2}\right)$. Un faisceautrayeur est branché sur le lactoduc au moyen d'une tubulure d'un diamètre intérieur de $12 \mathrm{~mm}$. Une pulsation simultanée (Vitesse : $60 \mathrm{p} / \mathrm{mn}$; rapport : 72,6 p. 100, a : 24,4 p. $100, b: 48,2$ p. $100, c: 13,7$ p. $100, d: 13,7$ p. 100) est transmise aux manchons par un relais électromagnétique situé à $1,50 \mathrm{~m}$ d'un régulateur à poids taré à $50 \mathrm{kPa}\left({ }^{3}\right)$ monté à l'extrémité de la ligne de vide.

(1) La ferminologie utilisée est celle de la norme française enregistrée U 36-000 d'obłobre 1975 : "Installations de traite mécanique. Vocabulaire."

* AFNOR. Tour Europe, 92080 Paris la Défense.

(2) $1 \mathrm{NI}$ : un litre d'air pris dans les conditions de l'atmosphère normale $\left(76 \mathrm{~cm} \mathrm{Hg} ; 20^{\circ} \mathrm{C}\right)$.

(3) 100 kilo Pascal $(\mathrm{kPa})=750$ millimètres de mercure $(\mathrm{mm} \mathrm{Hg})$. 
La griffe, d'un volume intérieur de $0,14 \mathrm{dm}^{3}$, ne possède pas de dispositif de coupure du vide. Le « lait » pénètre par 4 orifices de diamètre intérieur $7,3 \mathrm{~mm}$ et la sortie est reliée à un tuyau long à lait de $11 \mathrm{~mm}$ de diamètre intérieur.

Paramètres expérimentaux.

1. Débit d'écoulement du liquide. L'eau contenue dans un bocal de 25 litres s'écoule dans 4 trayons artificiels (fig. 1). Les débits de $1 \mathrm{l} / \mathrm{mn}$ et de $4 \mathrm{l} / \mathrm{mn}\left({ }^{1}\right)$ à travers le faisceau trayeur sont obtenus en fixant des ajutages de diamètres respectifs 1 ef $2 \mathrm{~mm}$ à la partie supérieure de chaque trayon.

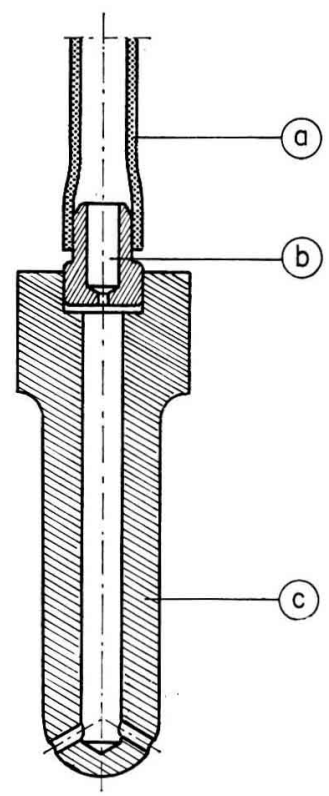

FIG. 1. - Schéma de trayon artificiel.

$a$ : tuyau en caoutchouc; $b$ : ajutage interchangeable permettant de limiter le débif ; $c$ : corps percé à la partie inférieure de 2 orifices permettant l'écoulement du liquide durant la phase d'ouverture du manchon.

2. Admission d'air à la griffe. L'air est admis à la partie supérieure de la griffe par un orifice de $0,78 \mathrm{~mm}$ de diamètre qui peut être aisément obstrué lorsque la traite s'effectue sans admission d'air.

3. Hauteur d'élévation du lait. Pour la traite en ligne basse, le lactoduc est situé à $0,08 \mathrm{~m}$ au-dessous de la sortie de la griffe et le tuyau long à lait a une longueur de $1,25 \mathrm{~m}$. En ligne haute, le lactoduc est élevé à 1,92 $\mathrm{m}$ au-dessus de la sortie de la

(1) Les valeurs mesurées sont de $1,0321 / \mathrm{mn}$ (erreur type de la moyenne : 0,024;32 données) et de $4,129 \mathrm{l} / \mathrm{mn}$ (erreur type de la moyenne : 0,$112 ; 32$ données). 
griffe et le tuyau long à lait à une longueur de 2,50 m. Dans les 2 cas, la pente du lactoduc vers la chambre de réception est d'environ 2 p. 100.

4. Type de manchon-trayeur. Les 2 manchons présentés à la figure 2 sont des prototypes Fullwood, type SL2, qui diffèrent seulement par la conception de la queue du manchon. Dans le modèle monobloc, le tuyau à lait $(\mathrm{di}=8 \mathrm{~mm} ; \mathrm{I}=155 \mathrm{~mm})$ est directement relié au corps tandis que dans l'autre type, une pièce de plastique dur sert à la fixation du tuyau.

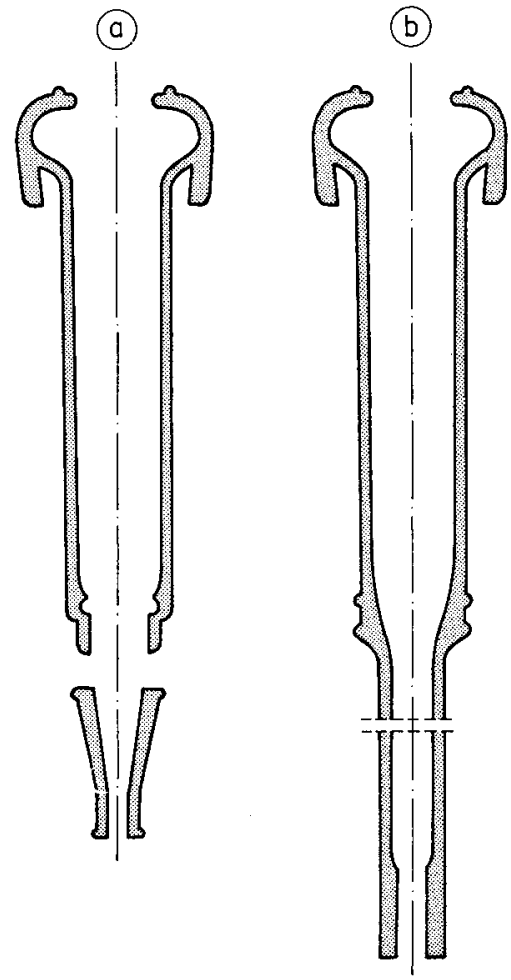

FIG. 2. - Schéma des manchons utilisées

$a$ : modèle composite pour lequel le tuyau à lait est relié au corps par l'intermédiaire d'un viseur en matière plastique ; $b$ : modèle monobloc.

\section{Enregistrements.}

Le déplacement de la paroi du manchon est enregistré à l'aide d'un capteur potentiométrique Pye-Ełher $\left(^{1}\right)$, type PD II scellé au gobelef à $40 \mathrm{~mm}$ au-dessous de l'extrémité du trayon artificiel.

Le vide sous le trayon est enregistré par un capteur de pression Endevco $\left({ }^{2}\right)$, type $8503+40$ Psi vissé à l'extrémité d'un tube d'acier inoxydable courbé $(\mathrm{di}=3 \mathrm{~mm}$,

(1) Représenté par Phymetron, 56, rue de la Bidassoa, 75020 Paris.

(2) Endevco, 76, rue des Grands-Champs, 75020 Paris. 
$I=40 \mathrm{~mm}$ ), qui pénètre sous le trayon en étant enfoncé à travers le manchon à la base de celvi-ci. Un capteur de même type permet l'enregistrement de la pulsation, en étant fixé directement au corps du gobelet.

Les signaux sont transmis à des ponts d'extensiométrie Sedeme $\left({ }^{1}\right)$, type TS 26, puis à un enregistreur oscillographique à trace directe Southern Instruments ( ${ }^{1}$ ), type 10400 sur lequel les tracés sont recueillis aux échelles suivantes : $100 \mathrm{~mm}$ pour une pression de $50 \mathrm{kPa}, 100 \mathrm{~mm}$ pour un déplacement de $10 \mathrm{~mm}$ et $220 \mathrm{~mm}$ pour un temps de 1 seconde.

\section{Paramètres mesurés.}

L'enregistrement simultané de la pulsation, du vide sous le trayon et du mouvement du manchon est analysé en mesurant les paramètres définis à la figure 3 .

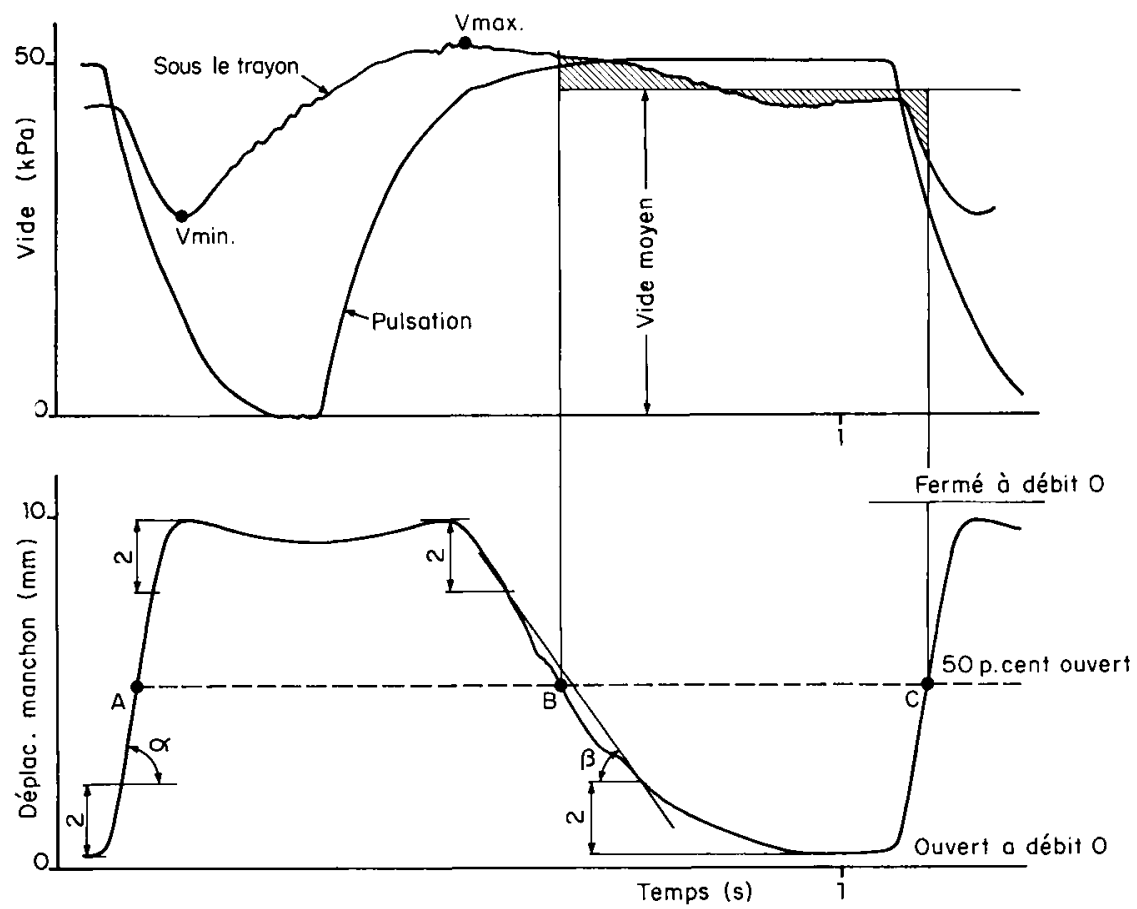

FIG. 3. - Définition des paramètres éfudiés.

En haut : enregistrement de la pulsation ef du vide sous le trayon ; en bas : enregistrement du mouvement du manchon. $V$ max et $\mathrm{V}$ min : vide maximum et minimum sous le trayon (en $\mathrm{kPa}$ ). Le rapport (en p. 100) d'ouverture du manchon, $\mathrm{Ro}=\frac{\mathrm{BC}}{\mathrm{AC}}$ correspond à la proportion du cycle de pulsation durant laquelle le manchon est plus qu'à moitié ouvert. Le vide moyen $(\mathrm{Vm})$ sous le trayon (en $\mathrm{kPa}$ ) est mesuré durant le temps où le manchon est plus qu'à la moitié ouvert. L'amplitude du déplacement du manchon (Am), en $\mathrm{mm}$, est égale à la distance parcourue par la paroi du manchon entre la position ouverte et la position fermée. La vitesse de fermeture (Vf) ef la vitesse d'ouverture ( $\mathrm{Vo}$ ), en $\mathrm{mm} / \mathrm{s}$ sont respectivement proportionnelles à la tangente de l'angle $\alpha$ et à celle de l'angle $\beta$.

(1) Représenté par Sedeme, 11, rue Simonet, 75013 Paris. 
Méthodes statistiques.

Les 4 facteurs contrôlés sont étudiés selon un plan factoriel : hauteur du lactoduc (ligne basse ou ligne haute), entrée d'air (avec ou sans admission d'air), type de manchon (monobloc ou en 2 parties). Pour chacune des 24 combinaisons factorielles, l'enregistrement est répété 4 fois ( 96 mesures au total).

Les effets principaux et les interactions sont testés en représentant chacune des 24 combinaisons par le résultat moyen des 4 répétitions factorielles. Les carrés d'interactions non significatives d'ordre supérieur à 2 sont considérés comme somme des carrés résiduelle (Vessereau, 1960).

\section{Résultats.}

Le vide sous le trayon.

- Le débit d'écoulement du lait est le principal facteur de perturbation du vide sous le trayon. Quelle que soit la hauteur du lactoduc (fig. 4) ou l'admission d'air à la griffe (fig. 5), $V$. min. décroît nettement tandis que $V$ max. demeure sensiblement constant lorsque le débit du lait s'accroît.

- En ligne haute, $V$ max et $V$ min sont plus faibles qu'en ligne basse (fig. $4 a$ et $b$ ). II en résulte que la fluctuation du vide ( $V$ max- $V$ min) bien que statistiquement plus faible en ligne basse $(P<0,01)$ demeure presque aussi importante qu'en ligne haute (fig. 4c).
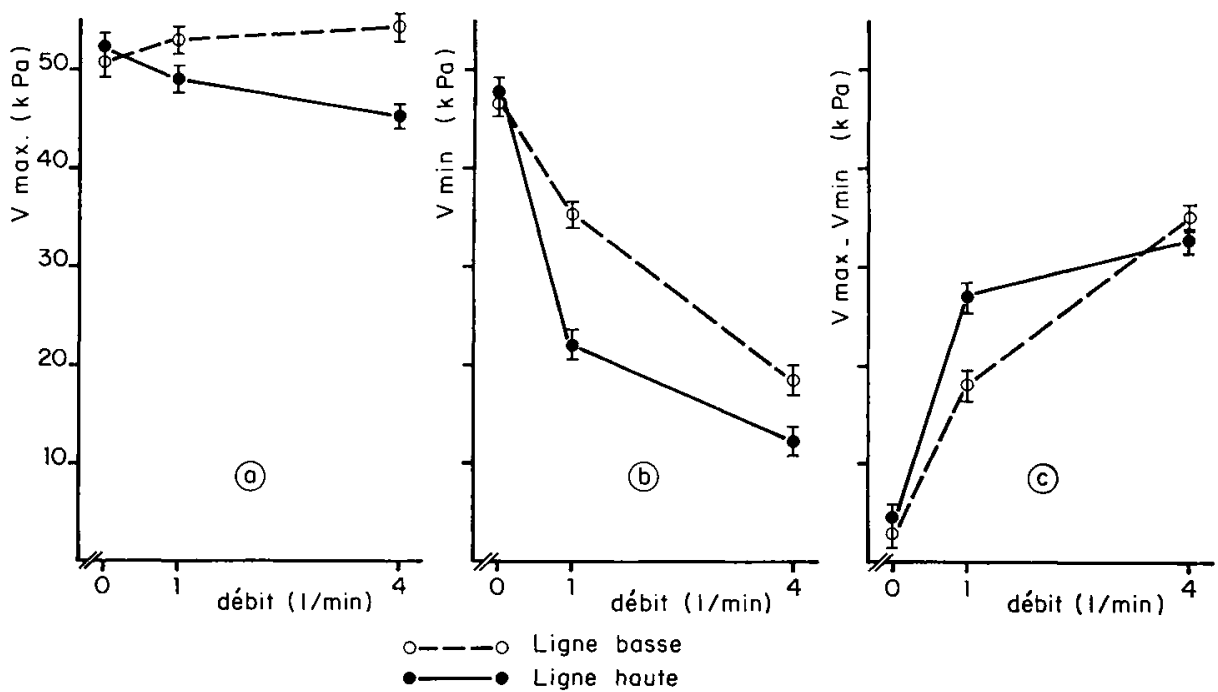

Ligne houte

FIG. 4. - Incidence de la hauteur du lactoduc sur la fluctuation du vide. Interactions (hauteur du lactoduc $\times$ débit) :

$a: V \max :$ Vide maximum $(P<0,05) ; b$ : Vmin : Vide minimum $(P<0,01)$; $c: V_{\max }-V_{\min }:(P<0,01)$. 
- L'entrée d'air, en limitani la diminution de $V \min$, réduit les fluctuations du vide (fig. $5 c)$ de façon nettement plus marquée $(P<0,01)$ que la ligne basse.
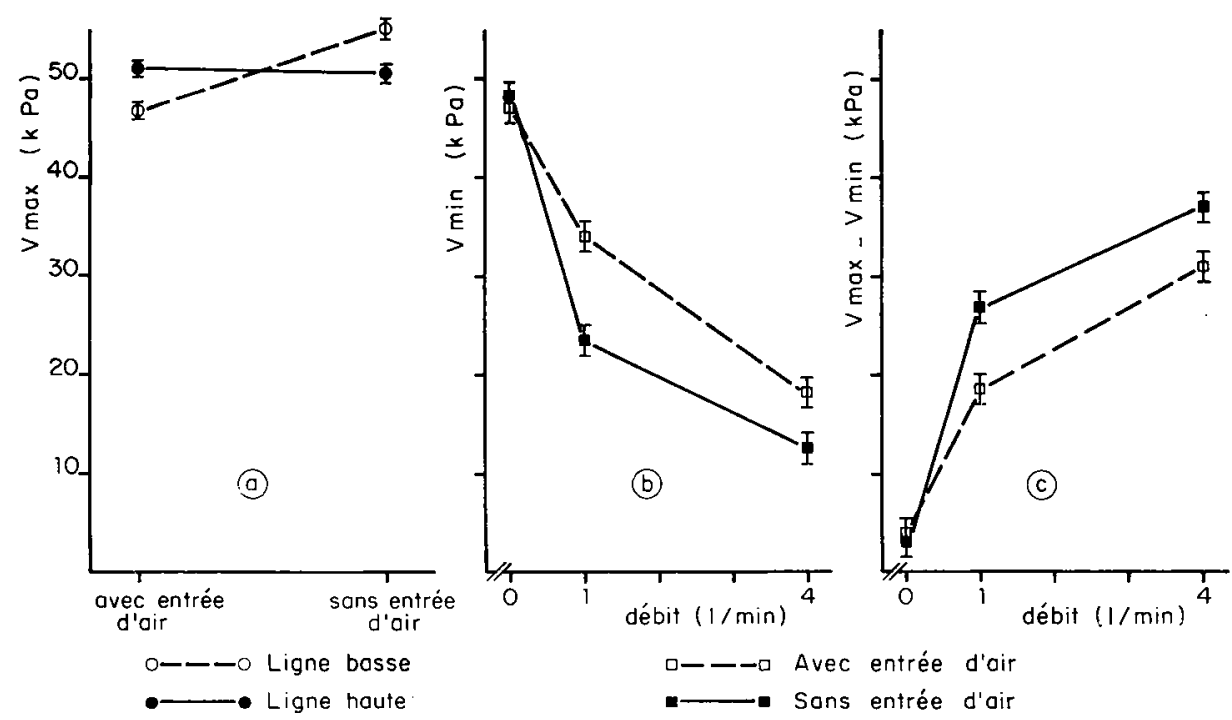

FIG. 5. - Incidence de l'entrée d'air sur la fluctuation du vide.

$a$ : Vmax : Vide maximum. Interaction (entrée d'air $\times$ hauteur du lactoduc) $(\mathrm{P}<0,01) ; b$ : Vmin : Vide minimum. Interaction (entrée d'air $\gamma$ débit) $(P<0,01) ; c: V$ max- $V$ min. Interaction (entrée d'air $\times$. débit) $(P<0,01)$.

De même, l'entrée d'air, sans effet sur $\vee$ max en ligne haute (moyenne $50,9 \mathrm{kPa}$ ), provoque une diminution significative de $\mathrm{V}$ max en ligne basse $(46,8 \mathrm{kPa}$ avec entrée d'air ; 54,7 kPa sans entrée d'air) (fig. 5a).

- Les interactions entrée d'air $x$ débit $x$ hauteur du lactoduc (fig. $6 a$ ) montrent qu'en ligne haute, le vide moyen sous le trayon est plus faible qu'en ligne basse. La différence est d'aułant plus grande que le débit du lait est élevé.

L'entrée d'air provoque une diminution de $\mathrm{Vm}$ en ligne basse et une augmentation en ligne haute. Il en résulte que le vide moyen sous le trayon peut être très différent du vide nominal mesuré au régulateur $(50 \mathrm{kPa})$. Ainsi, sans entrée d'air, $\mathrm{Vm}$ a pour valeur $51,4 \mathrm{kPa}$ en ligne basse ef $32,2 \mathrm{kPa}$ en ligne haute pour un débit du lait de $41 / \mathrm{min}$. La différence entre les deux systèmes est significativement atténuée lorsqu'on pratique une entrée d'air (respectivement $44,2 \mathrm{kPa}$ ef $38,8 \mathrm{kPa}$ ).

\section{L'ouverture du manchon.}

Avec l'entrée d'air, en ligne basse comme en ligne haute, le pourcentage du cycle durant lequel le manchon est plus qu'à demi-ouvert (Ro) n'est pas significativement affecté par l'écoulement de liquide et demeure voisin de sa valeur nominale mesurée pour un débit nul (Ro $=50$ p. 100) (fig. 6b). 
Sans entrée d'air, l'écoulement de liquide a pour effet d'accroître Ro en ligne haute $(P<0,01)$ et de diminuer Ro en ligne basse $(P<0,01)$. Il en résulte que le temps de demi-ouverture du manchon peut être différent du rapport de pulsation. Ainsi, pour un rapport de 72 p. 100 , aux débits de $1 \mathrm{l} / \mathrm{mn}$ ef $4 \mathrm{l} / \mathrm{mn}$, Ro prend les valeurs de 67,1 p. 100 et 86,1 p. 100 en ligne haute et de 41,9 p. 100 et 23,9 p. 100 en ligne basse.
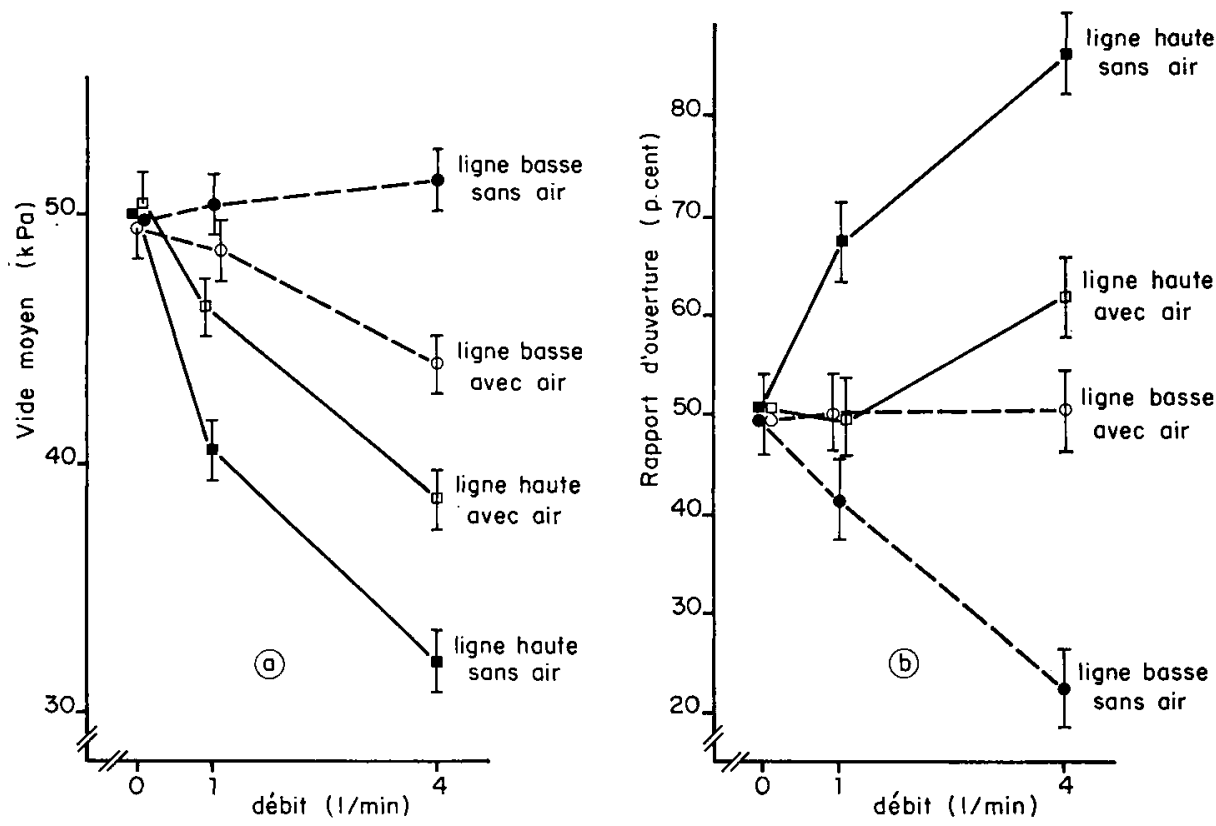

FIG. 6. - Variation du vide moyen sous le trayon et du rapport d'ouverture du manchon. Interactions (Débit $\times$ hauteur du lactoduc $X$ entrée d'air).

$a: V m$ : Vide moyen sous le trayon $(P<0,05) ; b$ : Ro : Rapport d'ouverture du manchon $(P<0,01)$

Enfin, la considération simultanée de Vm (fig. 6a) et de Ro (fig. 6b) montre que le temps durant lequel le manchon est plus qu'à moitié ouvert augmente quand le vide moyen sous le trayon diminue. Le manchon amorce son mouvement d'ouverture d'autant plus tard que $V m$ est élevé.

La vitesse de déplacement des parois du manchon.

L'écoulement du lait provoque une décroissance hautement significative $(P<$ 0,001 ) des vitesses d'ouverture et de fermeture du manchon (fig. 7 ). Le mouvement de fermeture s'amorce dès que le vide dans la chambre de pulsation commence à diminuer et s'effectue à vitesse constante (fig. 3). La vitesse de ce déplacement semble être d'autant moins élevée (fig. 7a) que le vide minimum sous le trayon est faible (fig. $5 b$ ). On remarque notamment que la vitesse de fermeture (fig. $7 a$ ) comme le vide minimum (fig. $5 b$ ) est accru avec entrée d'air et lorsqu'on dispose le lactoduc en ligne basse (Vf : $173,9 \mathrm{~mm} / \mathrm{s}$ contre $136,9 \mathrm{~mm} / \mathrm{s}$ en ligne haute) $(P<0,001)$. 

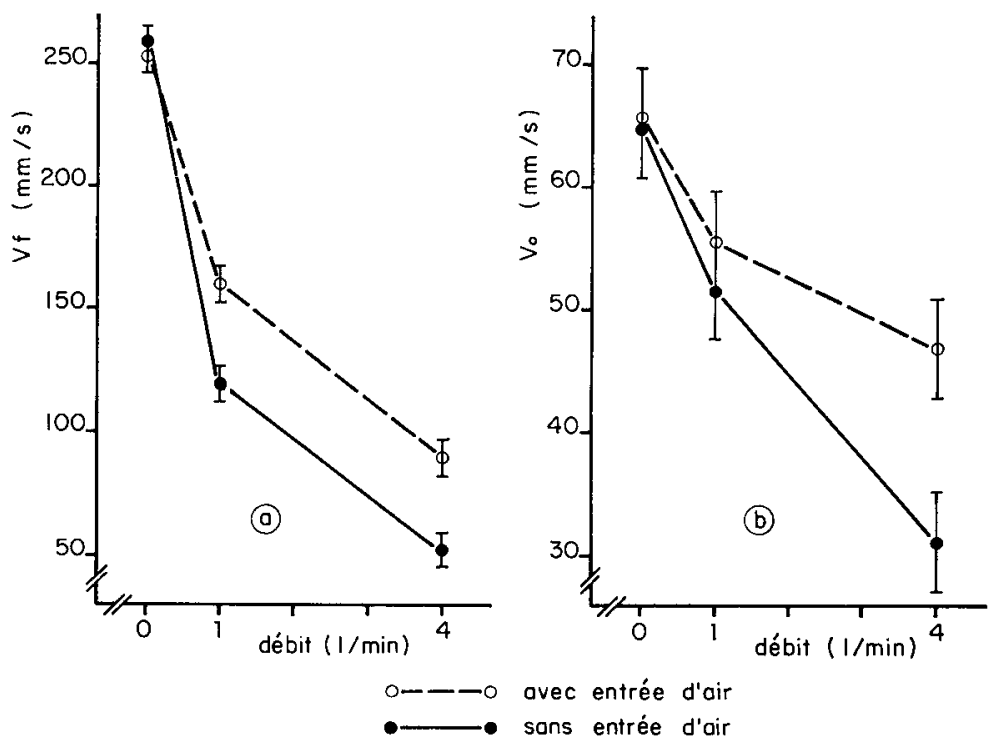

FIG. 7. - Evolution des vitesses de déplacement du manchon en fonction du débit. Interactions (débit $x$ entrée d'air).

$a:$ : $:$ Vitesse de fermeture $(P<0,01) ; b:$ Vo: Vitesse d'ouverture (Interaction non-significative. L'effet simple du débit est significatif au seuil 0,001).

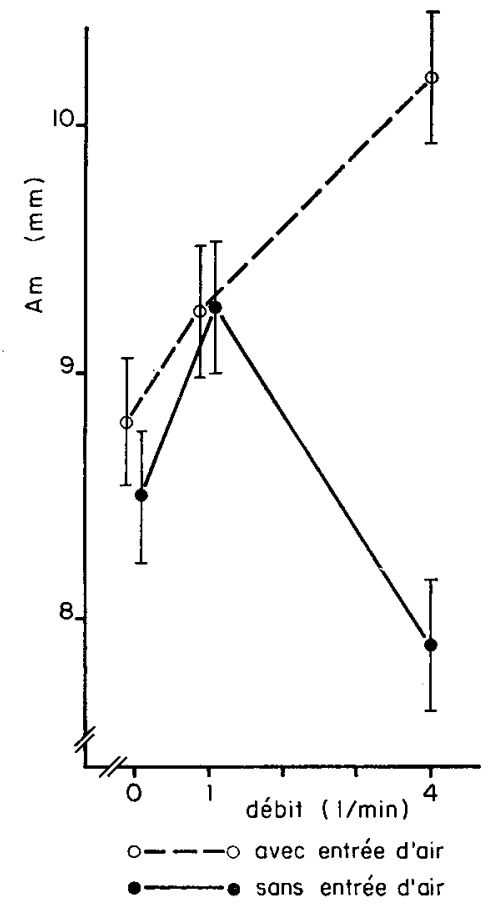

FIG. 8. - Incidence du débit sur l'amplitude (Am) du déplocement des"parois dümanchon. Interaction (entrée d'air $Y$ débit) significative au sevil 0,01. 
En contrepartie, l'ouverture du manchon qui se produit à vitesse moyenne moins grande que la fermeture (respectivement $250 \mathrm{~mm} / \mathrm{s}$ et $65 \mathrm{~mm} / \mathrm{s}$ au débit nul) semble difficilement prévisible à partir de la pulsation et du vide sous le trayon (fig. 3). On observe que le déplacement est d'autant plus ralenti que le manchon tend vers son ouverture maximale. Enfin, comme pour Vf, l'entrée d'air provoque un accroissement de $V o$ (à la limite de signification au seuil $P<0,05$ ) (fig. $7 b$ ).

L'amplitude du mouvement du manchon.

L'analyse de l'interaction entrée d'air $\times$ débit $(P<0,01)$ montre que l'amplitude $A_{m}$ du déplacement d'une paroi du manchon s'accroît (non significatif) lorsque le débit augmente de 0 à $1 \mathrm{l} / \mathrm{mn}$ (fig. 8). Ensuite :

Avec entrée d'air, l'accroissement de $A_{m}(P<0,01)$ se poursuit pour le débit le plus élevé $(4 \mathrm{l} / \mathrm{mn})$ jusqu'au ballonnement du manchon dont le diamètre devient alors plus important qu'au repos.

A l'inverse, sans entrée d'air, $A_{m}$ diminue au débit de $4 \mathrm{I} / \mathrm{min}$ et l'ouverture incomplète du manchon semble provenir de la diminution de Vo (fig. $7 b$ ).

\section{Discussion et conclusions.}

Effet perturbateur de l'écoulement du lait et adaptation de la machine à la physiologie de l'animal.

Les exigences physiologiques de l'animal étant actuellement très mal définies, nous sommes conduits à envisager deux hypothèses quant aux conditions de fonctionnement du manchon trayeur susceptibles d'être optimales.

Première hypothèse : Le fonctionnement du manchon est maintenu aussi proche que possible de celui qui existe en l'absence de débit du lait :

- L'écoulement du lait étant parmi les quatre facteurs que nous avons étudié celui qui modifie le plus le fonctionnement du manchon (diminution de la vitesse de déplacement des parois, accroissement des fluctuations du vide ainsi que perturbations du temps d'ouverture et du vide moyen sous le trayon), il peut apparaître souhaitable d'en minimiser l'effet, en maintenant des conditions de fonctionnement aussi proches que possible de celles qui sont constatées lorsque le débit du lai est nul.

On considère en général que :

- L'écoulement du lait provoque sous le trayon une fluctuation du vide (Noorlander et Schalm, 1958 ; Schalm et Noorlander, 1958 ; Whittlestone et Phillips, 1959 ; Maffey, 1961 ; Stanley, Kesler et Bortree, 1962; McDonald ef Witzel, 1968) dont l'amplitude croît avec le débit du lait (Thiel, Clough et Akam, 1964 ; Thiel et al., 1968 ; O'Callaghan, 1974). Le lait qui engorge le faisceau trayeur empêche alors la libre circulation de l'air dans celui-ci et nécessite un surcroît d'énergie pour être transporté (Thiel et al., 1968).

- Les bouchons de lait gênent le transit du lait entre le manchon et le volume collecteur (pot ou lactoduc) (Claesson, 1968 ; Schalm et Noorlander, 1958 ; Maffey, 1961) en provoquant, dans les cas extrêmes, l'engorgement total du manchon par le 
lait (McDonald et Witzel, 1968 ; Smith, 1970). On constate même que ces bouchons de lait peuvent avoir un mouvement d'oscillation périodique correspondant soit à la fréquence propre du système (Thiel, 1968) soit à une fréquence voisine de la vitesse de pulsation. II se produit alors des fluctuations du vide très importantes $(66 \mathrm{~cm} \mathrm{Hg}$ sur machine à traire pour brebis, Le Du, 1974, données non publiées).

- La conception du manchon peut influencer l'écoulement du laił. Ainsi, nous avons montré antérieurement que le manchon monobloc freine l'écoulement du lait car le tuyau de sortie s'applatit légèrement durant la phase de « massage ». La fluctuation du vide est alors nettement plus importante qu'avec le manchon en 2 parties : $65 \mathrm{~cm} \mathrm{Hg}$ contre $41 \mathrm{~cm} \mathrm{Hg}$ (pour une traite sans griffe, sans entrée d'air, avec un débit de $8 \mathrm{l} / \mathrm{min}$ à travers le faisceau trayeur). Nous ne confirmons pas ces résultats puisque dans nos conditions expérimentales le type de manchon n'a qu'une faible incidence sur le vide sous le trayon et le mode de déplacement de ses parois. Il est possible que les différences entre les manchons soient atténuées en utilisant une griffe d'un volume intérieur relativement grand $\left(0,14 \mathrm{dm}^{3}\right)$.

Deuxième hypothèse : Le fonctionnement du manchon est à chaque instant adapté au débit du lait qui le traverse.

Il est peut-être souhaitable que le fonctionnement du manchon varie avec le débit de lait qui le traverse afin d'optimiser à chaque instant la stimulation perçue par l'animal et maintenir des conditions satisfaisantes de décongestion du trayon. Dans ces conditions, le ralentissement des mouvements d'ouverture et de fermeture du manchon, observé également par Thiel, Clough et Akam (1964) et par O'Callaghan (1974) serait à interpréter en termes de diminution de l'énergie transmise au trayon sous forme cinétique par la masse du manchon en mouvement. Cette énergie, étant considérée comme un facteur important de stimulation et de décongestion (Zaks, 1962 ; Tröger et Lohr, 1967 ; Thiel, 1968 ; Brandsma, 1968), il conviendrait probablement d'atténuer la détérioration des caractéristiques dynamiques du manchon avec l'augmentation des débits.

Nous confirmons que durant l'écoulement du lait la fermeture du manchon s'amorce quand le vide de pulsation commence à diminuer tandis que l'ouverture est très décalée par rapport à la pulsation (Thiel, Clough et Akam, 1964 ; Guest, Shepardson et Towsend, 1965 ; O'Callaghan, 1974). Il en résulte que le mouvement du manchon, commandé à la fois par les variations de pression sous le trayon et dans la chambre de pulsation, ne peut être déduit de la courbe de pulsation (Hundtoft, 1962 ; Thiel, Clough et Akam, 1964).

La compréhension du fonctionnement de la machine à traire impose donc que l'on considère le mouvement réel du manchon. Dans ce contexte, un accroissement de l'amplitude de son mouvement $(\mathrm{Am})$ et du temps pendant lequel il est ouvert (Ro) nous semblent susceptibles de favoriser le transit d'un débit croissant de lait, à condition cependant que le vide moyen $(\mathrm{Vm})$ demeure suffisamment élevé.

Rôle de l'entrée d'air.

D'après nos résultats expérimentaux, l'admission d'air à la griffe offre une possibilité de régulation efficace : 
- Elle atténue les effets de l'augmentation des débits du lait (fluctuations du vide et diminution des vitesses de déplacement du manchon).

- En ligne haute comme en ligne basse, elle évite que le vide moyen, et le temps d'ouverture du manchon, ne s'écartent trop des valeurs nominales au débit nul.

- De plus, l'entrée d'air en accroissant l'amplitude du déplacement des parois du manchon et donc son volume intérieur en position ouverte est susceptible de faciliter l'évacuation du lait et d'éviter l'engorgement du manchon.

Il est admis que l'entrée d'air pratiquée au niveau de la griffe (Hopkirk, PalmerJones et Whittlestone, 1943 ; Whittlestone, 1948 ; Fincher, 1953) et plus rarement à l'embouchure des manchons (Gillies, 1903 ; Tolle, 1975 ; Tolle et Haman, 1975) ou à la base de ceux-ci (Smith, 1970) est une solution d'autant plus efficace pour faciliter l'écoulement du lait qu'elle se situe près de l'extrémité du trayon (McDonald ef Witzel, 1968). La réduction de l'ampliłude des fluctuations ne dépendrait pas du débit d'air admis (entre 2,8 ef $14 \mathrm{Nl} / \mathrm{min}$ à la griffe (Thiel et al., 1968).

Pour Claesson (1968) et Whittlestone (1968) l'entrée d'air serait indispensable en ligne haute. Toutefois, McDonald et Witzel (1968), signalant qu'elle accroît la lipolyse du lait, conseillent d'en réduire le débit au minimum nécessaire. Ceci serait facilité par une disposition du lactoduc en ligne basse (Whittlestone, 1968).

Incidence de la hauteur d'évacuation du lait.

La ligne haute (avec tuyau à lait de $2,50 \mathrm{~m}$ ) a principalement pour effet de faire décroître le vide moyen sous le trayon d'autant plus que le débit du lait est élevé. Cette disposition du lactoduc justifie l'emploi d'un vide nominal au régulateur plus élevé de 3 à $4 \mathrm{~cm} \mathrm{Hg}$ qu'en ligne basse de façon à ce que dans les deux cas, le vide moyen sous le trayon soit sensiblement identique (fig. 6a). La différence majeure entre les deux systèmes provient de ce qu'en ligne haute, lorsque le lait s'écoule, le vide nominal sous le trayon est diminué de la pression hydrostatique exercée par le mélange d'air et de liquide remplissant le long tuyau à lait disposé verticalement. Ainsi, pour une hauteur d'élévation donnée, on conçoit que la décroissance du vide sous le trayon est d'autant plus importante que la densité du mélange et donc le débit du lait sont élevés. De ce point de vue, quelque soit la hauteur d'élévation du lait, la fluctuation du vide proprement dite serait la conséquence de phénomènes de résonance dus à la pulsation (Phillips, 1965b) et dépendrait de la fréquence propre de la masse du faisceau trayeur en mouvement (Thiel, Clough et Akam, 1964) dont le laił ne représente qu'une faible part.

Etant donné qu'en augmentant la hauteur d'évacuation du lait, le vide maximum ef le vide minimum diminuent ensemble (fig. $4 a$ et $b$ ), l'accroissement global de la fluctuation du vide, en ligne haute, demeure peu important, bien qu'étant significatif (fig. 4c). Nos résultats confirment ceux de Claesson (1968) et Thiel et al (1968) obtenus également en laboratoire. Ainsi, Thiel ef al. (1968) enregistrent des fluctuations respectives de 6,$15 ; 6,40 ; 6,25 ; 5,80 ; 6,40$ pouces de mercure pour des accroissements successifs d'un pied de la hauteur du lactoduc.

II faut noter que Mein (1967) qui observe une fluctuation du vide nettement plus important en ligne haute $(96 \mathrm{~mm} \mathrm{Hg})$ qu'en ligne basse $(38 \mathrm{~mm} \mathrm{Hg})$ effectue ses mesures à la griffe et non pas directement sous le trayon. Enfin, les salles de traite en 
ligne basse éfudiées lors des enquêtes en ferme sont souvent d'installation plus récente que celles en ligne haute et possèdent des lactoducs de diamètre très important $(76 \mathrm{~mm}$ et $89 \mathrm{~mm}$, selon Fell, 1969).

Or, l'accroissement du volume récepteur du lait suffit à améliorer la stabilité du vide (Noorlander et Schalm, 1958 ; Schalm, Noorlander et Pier, 1957).

Durant la traite, les différences enregistrées en faveur de la ligne basse sont :

- une diminution du temps de traite (Mein, 1967, 1968) : 48 secondes $(P<0,01$ ) lors d'une enquête en ferme et 36 secondes à la suite d'une étude portant sur 17 animaux ;

- un accroissement des débits moyens et maximum d'écoulement du lait (Mein, 1967, Clarke et Lascelles, 1965, 1966 ; Phillips, 1969).

Toutefois, ces effets bénéfiques seraient dus au vide nettement plus élevé mesuré sous le trayon ou à la griffe lorsqu'on trait en ligne basse : $381 \mathrm{~mm} \mathrm{Hg}$ et $320 \mathrm{~mm} \mathrm{Hg}$ selon Mein (1967); $302,3 \mathrm{~mm} \mathrm{Hg}$ et $256,5 \mathrm{~mm} \mathrm{Hg}$ selon Clarke et Lascelles (1966). En fait, l'accroissement du débit en ligne basse est compensé par un égouttage moins rapide (Phillips, 1969) et plus important (Clarke et Lascelles, 1965 cité par Woolford, 1974) tandis que simultanément, le phénomène de grimpage des gobelets le long des trayons, en général, considéré comme nocif, serait intensifié (Clarke et Lascelles, 1965 ; Phillips et Copeman, 1969). Il en résulte que le temps de traite total n'est pas affecté. On admet, par conséquent, que la hauteur du lactoduc n'a aucune incidence réelle sur la productivité horaire du trayeur (Mein, 1967 ; Fell, 1969).

Conséquences globales.

L'écoulement du lait étant le facteur principal de perturbation au niveau du manchon, nos résultats :

- confirment la nécessité de contrôler les installations à la ferme durant la traite et non pas, comme actuellement, en l'absence des animaux ;

- limitent l'intérêt des tentatives actuelles de normalisation du matériel effectuées en l'absence de données scientifiques rigoureuses.

En effet, il apparaît indispensable d'élaborer une théorie qui interprêterait le fonctionnement de la machine à traire en terme de transfert d'énergie au trayon en tenant compte de ses caractéristiques biologiques. Or, celles-ci sont méconnues et les modèles tant mécanique de Phillips (1965a) que mathématique de Towsend (1969) sont à cet égard rudimentaires.

Par ailleurs, la cinématique du mouvement du manchon propre à « stimuler » et à « décongestionner » demeure fout aussi méconnue et demanderait au moins à être abordée par l'étude des pressions appliquées directement par le manchon au trayon. Outre, une conception plus rationnelle du matériel, ce contexte devrait aussi permettre de cerner avec plus d'équité la part impartie à la machine à traire dans les expérimentations à caractère zootechnique concernant non seulement la traite proprement dite mais également l'éjection du lait et la sélection au regard de l'aptitude à la traite. 


\section{Références}

BRANDSMA S., 1968. Stimulation by the milking machine. Proc. Symp. Machine Milking, Nat. Inst. for Res. in Dairying, 119-129.

CLAESSON O., 1968. The significance of vacuum fluctuation in relation to udder health. Proc. nat. Mastitis Counc., Feb. 15-16, Chicago.

CLARKE D. J., LASCELLES A. K., 1965. Factors affecting the rate of milk flow in pipeline machines. Aust. J. exp. Agric. Anim. Husb. 5, 115-119.

CLARKE D. J., LASCELLES A. K., 1966. Cité par FELL L. R., 1969.

FELL L. R., 1964. Machine milking and mastitis : a review. Dairy Sci. Abstr., 26, 551-560.

FELL L. R., 1969. Lowline herringbone sheds. Vic. Dairy Farming Dig. 16, 2-7.

FINCHER M. G., 1953. Etiology, prevention and treatment of bovine mastitis. Proc. 15th Inst. Vet. Congr., Part. II, 122-145.

GUEST R. W., SHEPARDSON E. S., TOWNSEND J. S., 1965. Engineering aspects of milking machine research. J. Milk Fd Technol., 28, 166-169.

GILLIES A., 1903. Brit. Pat. 12958.

HOPKIRK C. S. M., PALMER-JONES T. WHITTLESTONE W. G., 1943. The effect of closed air admission on the health of the udder of dairy cows. N. Z. J. Agric., 66, 30-34.

HUNDTOFT E. B., 1962. Thèse non publiée. Agric. Eng. Depart., Cornell. University, Ithaca. Cité par GUEST R. W., SHEPARDSON E. S., TOWNSEND J. S. (1965).

LABUSSIERE J., RICHARD Ph., 1965. La traite mécanique. Aspects anatomiques, physiologiques et technologiques. Mise au point bibliographique. Ann. Zootech., 14, 63-126.

LE DU J., 1974. Particularité du fonctionnement du manchon-trayeur pour brebis (données non publiées).

MCDONALD J. S., WITZEL D. A., 1968. Vacuum fluctuation at the teat end during mechanical milking. J. Dairy Sci., 51, 543-548.

MAFFEY J., 1961. The physical principles of the milking machine. Vet. Rec., 73, 589-600.

MEIN G. A., 1967. Lowline milking. Vic. Dairy Farming Dig., 14, 8-12.

NOORLANDER D. O., SCHALM O. W., 1958. A method for graphic measurement of the physical forces and differential pressures in mechanical milking systems. J. amer. vet. med. Ass., 133, 474-479.

NYHAN J. F., 1968. The effect of vacuum fluctuation on udder disease. Proc. Symp. Machine Milking. Nat. Inst. for Res. in Dairying, 71-82.

O'CALLAGHAN E., 1974. Developpement of techniques for measuring four parameters in milking machines. Ir. J. agric. Res., 13, 323-333.

PHILLIPS D. S. M., 1965a. The theory of milking action at the cow's teat. Proc. Third Summer School and Conference on Mochine Milking, Hobart, 34-57.

PHILLIPS D. S. M., 1965b. The effect of pulsator rate on vacuum variations at the teat cup. Proc. Third Summer School and Conference on Machine Milking., Hobart, 103-105.

PHILLIPS D. S. M., 1969. Milking machine design and installation. Proc. N. Z. School and Conference on Machine Milking, 85-93.

PHILLIPS D. S. M., COPEMAN P. J. A., 1969. Some recent research on milking machine design. Proc. N. Z. Soc. anim. Prod., 29, 26-36.

SCHALM O. W., NOORLANDER D. O., PIER A. C., 1957. Mechanical milking and mastitis. Proc. U. S. Livestock Sanitary Ass., 187-196.

SCHALM O. W., NOORLANDER D. O., 1958. The milking machine teat cup in relation to the mastitis problem. Southwest. Vet., 11, 124-128.

SMITH F. F., 1970. A study of teatcup flooding. Southern California Dairy Cattle Day., Univ. California, Riverside, 51-55.

STANLEY D. E., KESLER E. M. BORTREE A. L., 1962. Effect of a fluctuating milking vacuum on certain measures of udder health. J. Dairy Sci., 45, 1343-1347.

THIEL C. C., CLOUGH P. A., AKAM D. N., 1964. Mechanics of machine milking. I. Pressures in the teatcup assembly and liner wall movement. J. Dairy Res., 31, 303-313.

THIEL C. C., 1968. Forces acting on the teat. Proc. Symp. Machine Milking., Nat. Inst. for Res, in Dairying, 3-14. 
THIEL C. C., CLOUGH P. A., WESTGARTH D. R., AKAM D. N., 1968. Factors affecting vacuum within the teatcup liner during milking. J. Dairy Res., 35, 303-316.

THIEL C. C., COUSINS C. L., WESTGARTH D. R., REITER B. 1969. Impact force as a possible cause of mechanical transfer of bacteria to the interrior of the cow's teat. J. Dairy Res., 36, 279-297.

TOLLE A., 1975. German Federal Patent Application no 2332355.

TOLLE A., HAMAN J., 1975. Milking without pulsation by means of a jacketed air-flow cushion in single-chamber teat cups (PME-System). Doc. 29 Session III. 10 PP. I. D. F. ; Mastitis Control Seminar, Reading.

TRÖGER F., LOHR H., 1967. Verfahren zur Mechanisierung des Anrüstens (Eutermassage) beim Melken der Kühe mił der Melkmaschine. Tierzucht, 21, 184-188.

TOWSEND F. S., 1969. A mathematical modeling approach to the study of the mechanics of milking, Thèse Agric. Eng. Dept., Cornell Univ., Ithaca, 89 pp.

VESSEREAU A., 1960. Méthodes statistiques en biologie et en agronomie, Vol. 2, 251-252. Baillière et Fils, Paris.

WHITTLESTONE W. G., 1948. Efficient mechanical milking. Austr. J. Dairy Technol., 3, 45-72.

WHITTLESTONE W. G., PHILLIPS D. S. M., 1959. Machine milking of dairy cows. Bull. $n^{\circ} 260$, N. Z. Department of Agriculture.

WHITTLESTONE W. G., 1968. Effects of milking machines on the quality of milk. J. Milk Fd. Technol., 31, 74-77.

WHITTLESTONE W. G., JONES M. A. S., 1969. Contamination of the bovine streak canal during milking. N. Z. vet. J., 17, 181-182.

WITZEL D. A., MCDONALD J. S., 1964. Bovine intramammary pressure changes during mechanical milking. J. Dairy Sc., 47, 1378-1381.

WOOLFORD M. W., 1974. Milking machines. 5th Austr. Milking Conf., Hawkesbury Agric. Coll. Lecture 7, 32 pp.

ZAKS M. G., 1962. The motor apparatus of the mammary gland, 188 pp., Oliver and Boyd, Londres. 